\title{
MARIA CECÍLIA PUNTEL DE ALMEIDA: A TRAJETÓRIA DE UMA PROTAGONISTA DA ENFERMAGEM BRASILEIRA
}

\author{
Silvana Martins Mishimaㄹ, Cinira Magali Fortuna², Carmen Gracinda Silvan Scochi', Maria José Bistafa \\ Pereira ${ }^{4}$, Regina Aparecida Garcia de Lima ${ }^{5}$, Silvia Matumoto ${ }^{6}$
}

${ }^{1}$ Livre-Docente em Planejamento e Gestão em Saúde. Professor Titular do Departamento de Enfermagem Materno-Infantil e
Saúde Pública (DEMISP) da Escola de Enfermagem de Ribeirão Preto (EERP) da Universidade de São Paulo (USP). São Paulo,
Brasil. E-mail: smishima@eerp.usp.br
${ }^{2}$ Doutora em Enfermagem em Saúde Pública. Professor Doutor do DEMISP da EERP/USP. São Paulo, Brasil. E-mail: fortuna@
eerp.usp.br
${ }^{3}$ Livre-Docente em Enfermagem Pediátrica e Neonatal. Professor Titular do DEMISP da EERP/USP. São Paulo, Brasil. E-mail:
cscochi@eerp.usp.br
${ }^{4}$ Livre-Docente em Planejamento e Gestão em Saúde. Professor Associado do DEMISP da EERP/USP. São Paulo, Brasil. E-mail:
zezebis@eerp.usp.br
${ }^{5}$ Livre-Docente em Enfermagem Pediátrica. Professor Titular do DEMISP da EERP/USP. São Paulo, Brasil. E-mail: limare@
eerp.usp.br
${ }^{6}$ Doutora em Enfermagem em Saúde Pública. Professor Doutor do DEMISP da EERP/USP. São Paulo, Brasil. E-mail:
smatumoto@eerp.usp.br

RESUMO: O presente artigo de reflexão tem por objetivo relacionar a trajetória da Profa. Dra Maria Cecília Puntel de Almeida com a história da enfermagem mais recente, apontando suas contribuições para a compreensão da enfermagem como trabalho. Nos últimos quarenta anos dedicou seus estudos e sua prática para a formação de pesquisadores de enfermagem, para construção da reforma sanitária brasileira, para a consolidação de uma perspectiva política da enfermagem brasileira no processo de construção de uma prática de saúde e de enfermagem mais democrática e para a formação de enfermeiros engajados em mudanças das práticas de enfermagem e dos serviços de saúde. Suas produções constituem uma demarcação da enfermagem como resultado e resultante das relações históricas, sociais, econômicas e culturais. Concluímos que seu legado é produto e produtor do movimento de engajamento da enfermagem brasileira em lutas pela democracia e pela saúde como direito.

DESCRITORES: História da enfermagem. Enfermagem em saúde pública. Sistema Único de Saúde.

\section{MARIA CECÍLIA PUNTEL DE ALMEIDA: THE TRAJECTORY OF A BRAZILIAN NURSING PROTAGONIST}

\begin{abstract}
The objective of this reflective paper is to relate the trajectory of Prof. Dr. Maria Cecília Puntel de Almeida with the most recent history of nursing, pointing out her contribution to the comprehension of nursing as work. Over the last forty years she has dedicated her study and practice to preparing nursing researchers, to building Brazilian healthcare reform, to consolidating a political perspective of Brazilian nursing in the process of building healthcare and democratic nursing practice, and to preparing nurses to be involved with changes in nursing practice and healthcare services. Her publications constitute a nursing benchmark as a result of historical, social, economical and cultural relationships. We conclude that her legacy is the product and a producer of the Brazilian nursing involvement movement in the causes of democracy and healthcare as a right.
\end{abstract}

DESCRIPTORS: History of nursing. Nursing in public healthcare. Brazilian Healthcare System.

\section{MARIA CECÍLIA PUNTEL DE ALMEIDA: LA TRAYECTORIA DE UNA PROTAGONISTA DE LA ENFERMERÍA BRASILEÑA}

RESUMEN: El presente artículo de reflexión tiene como objetivo relacionar la trayectoria de la Profa. Dra. Maria Cecília Puntel de Almeida con la historia más reciente de la enfermería, mostrando sus contribuciones para la comprensión de la enfermería como trabajo. En los últimos cuarenta años ha dedicado sus estudios y su práctica a la formación de investigadores en enfermería, a la construcción de la reforma sanitaria brasileña, a la consolidación de una perspectiva política de la enfermería brasileña en el proceso de construcción de una práctica de salud y de enfermería más democrática y a la formación de enfermeros involucrados en las transformaciones de las prácticas de enfermería y de los servicios de salud. Su producción constituye una demarcación de la enfermería como resultado y resultante de las relaciones históricas, sociales, económicas y culturales. Concluimos que su legado es producto y productor del movimiento de participación de la enfermería brasileña en luchas por la democracia y por la salud como derecho.

DESCRIPTORES: Historia de la enfermería. Enfermería en salud pública. Sistema Único de Salud. 


\section{INTRODUÇÃO}

O presente artigo tem por objetivo relacionar a trajetória da Profa. Dra. Maria Cecília Puntel de Almeida com a história da enfermagem mais recente, apontando suas contribuições para a compreensão da enfermagem como trabalho. Assim propomos uma pequena aproximação à trajetória da Profa. Dra. Maria Cecília para que, conhecendo um pouco de seu trabalho, possamos conhecer uma parte da história da enfermagem brasileira.

Um primeiro ponto, e que nos aproxima de Cecília, é olhar para sua contribuição para com a enfermagem brasileira estudando-a como trabalho e como prática social. Nos seus dizeres, "O trabalho é uma atividade inalienável do ser homem, mas comporta em si um paradoxo, de um lado traz uma visão negativa, uma visão de sobrevivência, dor e sofrimento, por exemplo, o trabalho de parto, o trabalho escravo, o trabalho explorado. Esta visão também está relacionada à alienação, ou seja, o homem não se reconhece enquanto produtor da sociedade, não se reconhece no produto que constrói, ou não tem acesso a esses bens produzidos, ou perde o conhecimento de todo o processo de trabalho e do significado das relações dos trabalhos entre si. Por outro lado tem uma visão positiva, de emancipação e de criatividade e de alteridade. Através do trabalho passamos a conhecer o outro e assim a nos conhecer". .

Neste sentido, ao retomarmos sua compreensão do trabalho na sociedade contemporânea, podemos buscar conhecer a trajetória de Cecília enquanto um ator social, que, com outros parceiros, se fez presente na construção da enfermagem brasileira.

\section{A TRAJETÓRIA DE FORMAÇÃO E O TRA- BALHO EM EQUIPE}

A Profa. Cecília, como era carinhosamente chamada por todos que a conheciam, foi Professora Titular do Departamento de Enfermagem MaternoInfantil e Saúde Pública da Escola de Enfermagem de Ribeirão Preto (EERP) da Universidade de São Paulo (USP), com dedicação integral ao trabalho de docência e pesquisa de 1968 a 2008, quando por motivos de saúde, teve que se aposentar desta instituição. A Profa. Cecília graduou-se em Enfermagem na EERP/USP em 1963; obteve os títulos de mestre e doutor em Saúde Pública pela Escola Nacional de Saúde Pública (ENSP/FIOCRUZ-Rio de Janeiro), respectivamente em 1967 e 1984.

Em 1991, submeteu-se, com êxito, a concurso para obtenção do título de Livre-Docente junto ao Departamento de Enfermagem Materno-Infantil e Saúde Pública da EERP/USP. O ápice da carreira foi conquistado em 1993, por meio da aprovação em concurso para provimento de cargo de Professor Titular lotado no mesmo Departamento.

Cabe destacar que seu mestrado intitulado "A enfermagem de saúde pública na unidade sanitária", foi realizado quando ainda era enfermeira de Saúde Pública junto ao Hospital das Clínicas de Ribeirão Preto, por incentivo do então Departamento de Medicina Preventiva da Faculdade de Medicina de Ribeirão Preto (FMRP) da USP. Cecília comentou em seu Memorial para o concurso de Professor Titular da EERP/USP que o "fato de ter realizado o mestrado em outra unidade, fora de Ribeirão Preto e com outros profissionais de saúde pública [...] permitiu dar início a uma atividade profissional com uma visão mais geral dos problemas de saúde e a importância da multidisciplinaridade para tratar destes problemas" ${ }^{1: 3}$

A compreensão de que os problemas de saúde ultrapassam as barreiras e delimitações das profissões e que o cuidado reveste-se de uma complexidade que clama por diversos saberes e fazeres estão presentes nas produções cientificas da Profa. Cecília e nas produções da enfermagem contemporânea. Ela discutiu o trabalho em equipe e a interdisciplinaridade como aspectos relevantes para o cuidado de enfermagem.

Se nota que as produções sobre o trabalho em equipe para a enfermagem até então, tinham por base a escola de relações humanas de Elton Mayo, que voltavam à atenção para o trabalhador e para a satisfação no trabalho visando a maximização dos resultados que se traduzem no aumento de produtividade. $^{2}$

A perspectiva da enfermagem como prática social considera a divisão técnica e social do trabalho, afirmando que não se trata apenas de uma divisão de funções e tarefas entre os diversos trabalhadores da enfermagem, mas uma divisão social de saberes e poderes que materializa a divisão de classes sociais no cotidiano do trabalho. ${ }^{2-3}$ Esse é certamente um importante atravessador do traba-

Almeida MCP. O processo de trabalho da enfermagem nos serviços de atenção básica em saúde. Aula ministrada no mestrado em enfermagem da Universidade Federal do Mato Grosso, Faculdade de Enfermagem em 21 de Maio de 2007. Disponível em documento Word [material não publicado] 
lho em equipe. Assim, o trabalho em equipe não poderia ser considerado como algo dependente individualmente das pessoas e da boa vontade destas, mas como uma complexidade que envolve conflitos e uma importante divisão social, resultante do modo de produção capitalista. ${ }^{4}$

Em uma de suas produções bibliográficas, ${ }^{5}$ apresenta junto com uma de suas parceiras de produção por longos anos, ${ }^{* *}$ o trabalho da enfermagem em saúde coletiva e a interdisciplinaridade. As autoras concluem o estudo dizendo que "o conhecimento capaz de fundamentar o cuidado de enfermagem deve ser construído na intersecção entre a Filosofia, que responde a grande questão existencial do homem, a Ciência e Tecnologia tendo a lógica formal como responsável pela correção normativa, e a Ética, isto é, numa abordagem epistemológica efetivamente comprometida com a emancipação humana". 4:100

Outro aspecto importante que merece ser destacado, é que este processo de Cecília prepararse em outra Universidade se deu atrelado também ao fato de que na época existiam poucos cursos de pós-graduação na área de enfermagem, sendo que o curso de doutorado em Saúde Pública da Escola Nacional de Saúde Pública (RJ), cursado por ela, foi criado em 1980, com enfoque multiprofissional, "dando um espaço às ciências sociais para contribuírem na compreensão das práticas de saúde". 2:19

Assim, o doutorado, também realizado na Escola Nacional de Saúde Pública (ENSP), permitiu que Cecília construísse outras parcerias, como por exemplo, com os professores Maria Cecília Donangelo, Ricardo Bruno Mendes Gonçalves, expoentes da Saúde Coletiva brasileira, que se estenderiam para a construção de outros espaços de produção.

\section{OS MOVIMENTOS SOCIAIS, A ENFER- MAGEM BRASILEIRA E A REFORMA SANITÁRIA BRASILEIRA}

Seu doutorado "Estudo do saber de enfermagem e sua dimensão prática", defendido em 1984, foi um marco para a enfermagem brasileira, uma vez que traduziu questões importantes acerca da compreensão da prática de enfermagem, em um contexto específico, o Brasil, em consonância com o movimento inicial presente na enfermagem brasileira principalmente a partir da década de 1970.
$\mathrm{Na}$ apresentação de seu estudo, publicado posteriormente sob o mesmo título, Cecília nos aponta que a "produção não é um resultado individual, mas está intimamente ligada às circunstâncias e pessoas com quem cruzamos e compartilhamos momento, idéias, leituras, numa discussão aberta e não mítica das questões da enfermagem brasileira". 2:19

Nas décadas de 1970 e início dos anos 1980, a produção científica da enfermagem estava marcada com temáticas em que predominavam aspectos internos da profissão como tecnologias aplicadas na assistência, elaboração de modelos assistenciais, avaliação de cuidados biopsicossociais, dentre outros, ${ }^{2}$ ou seja, a " preocupação é com a internalidade destes serviços e com as relações interpessoais dos agentes, sem referência à totalidade de que fazem parte, mas sim visando a racionalidade dos serviços e a eficácia dos agentes" 2:22

A produção de Cecília demarca outro patamar de produção científica que se junta ao movimento social presente no Brasil, incluindo o de saúde com um deslocamento para a busca de relações entre a área da saúde, a estrutura social e o modo de produção capitalista. ${ }^{5}$ É na década de 1980 também, que atores sociais ligados às universidades, aos movimentos estudantis, aos sindicatos, movimentos eclesiais e de morados se aglutinam em torno da reforma sanitária brasileira. ${ }^{6}$

Seus principais fundamentos defendidos foram a saúde como direito de cidadania e dever do Estado, considerando-a intrinsecamente relacionada à estrutura social. Na oitava Conferência Nacional de Saúde essa é conceituada como "resultante das condições de alimentação, habitação, educação, renda, meio ambiente, trabalho, transporte, emprego, lazer, liberdade, acesso e posse da terra e acesso a serviços de saúde. É assim, antes de tudo o resultado das formas de organização social da produção, as quais podem gerar grandes desigualdades nos níveis de vida" ,7:382 a Saúde não é um conceito abstrato. Define-se num contexto histórico de determinada sociedade e, num dado momento de seu desenvolvimento, devendo ser conquistada pela população em suas lutas cotidianas.

Podemos dizer que nesse momento da história recente do Brasil algumas enfermeiras organizadas constituíram-se em ator social nesse jogo de disputa pela saúde como direito. Ator social é

* Esta parceria refere-se à companheira de trabalho na EERP/USP - Professora Doutora Semíramis Melo Rocha, uma das Presidentes do Centro de Estudos e Pesquisa em Enfermagem. Com a professora Semíramis, Cecília dividiu por vários anos a coordenação do Núcleo de Pesquisas e Estudos em Saúde Coletiva. 
aquele que porta projetos e participa de um jogo social sendo capaz de produzir fatos novos nesse jogo. ${ }^{8}$ Assim, teve-se neste momento, enfermeiras que faziam parte da Associação Brasileira de Enfermagem (ABEn), de movimentos sindicais, enfim, um conjunto de atores que se colocaram na disputa para a construção de um projeto de sociedade mais democrática e justa.

A Profa. Cecília foi partícipe desse movimento que pode construir novos rumos para as políticas de saúde no país com a instituição do Sistema Único de Saúde, e com a possibilidade de se estabelecer outras bases para o trabalho de enfermagem. ${ }^{9}$

Cabe destacar que, a enfermagem brasileira através da $\mathrm{ABEn}$, neste momento, se constituiu um ator social importante, e só o fez pelo movimento de análise e revisão que se traduziu à época pelo denominado Movimento Participação que se tratou de "um Movimento Social da Enfermagem que transformou o modus operandi e a concepção política da ABEn". 10:229

O Movimento Participação se traduziu por um processo amplo de "trabalhadores de enfermagem e lideranças que realizavam uma análise da situação da enfermagem brasileira abordando questões como: a identidade profissional; a composição da força de trabalho; organização e relações de trabalho; o papel político da categoria na sociedade; a visão sobre saúde e a realidade institucional do setor; a fragilidade da representação da profissão fragmentada e com pouca visibilidade $[\ldots] "$, 10:229 incitando à reflexão da mudança de postura mais pró-ativa da ABEn em relação às questões da democracia e das políticas governamentais.

Neste cenário, merece ser destacada a atuação da Profa. Cecília, que por vários anos pode colaborar com a ABEn nestas questões. Cecília assumiu, em três diferentes gestões, a função de vice presidente no Distrito de Ribeirão Preto - Seção São Paulo. Sua participação nacional se deu como membro do Centro de Estudos e Pesquisas em Enfermagem (CEPEn), de 1977 a 1989. Durante este período foi Diretora do CEPEn e membro da Diretoria da ABEn Central.

Cabe destacar sua participação nestes espaços, colaborando de forma intensa na definição dos rumos desta Associação. Sua presença foi marcante na organização e definição dos Congressos Brasileiros de Enfermagem, principalmente na década de 1980, quando a enfermagem encontra-se imersa nesta reflexão sobre sua organização e seu proces- so de trabalho. Em Florianópolis, durante o $41^{\circ}$ Congresso Brasileiro de Enfermagem participou do grupo que desenvolveu três dos temas Oficiais do Congresso: Situação da enfermagem Brasileira na década de 80, O conhecimento técnico científico da Enfermagem na década de 80 e os Desafios da Enfermagem para a década de $80 .{ }^{11-13}$

Mesmo correndo o risco de não citar todas as parceiras desta época, vale lembrar de: Ieda Barreira, Semíramis Melani Melo Rocha, Maria Tereza Leopardi, Maria José dos Santos Rossi, Briguita Castellanos, Emília Luigia Saporiti Angerami.

Cecília escreveu que a ABEn foi a "Possibilidade de junto com os pares da profissão batalhar por mudanças na enfermagem e no setor saúde, significa a possibilidade de desenvolver um trabalho coletivo, caminho este em que acredito". 1:220 Crença que a levou a trabalhar de forma contínua, compromissada, responsável e ética junto à enfermagem e à saúde, dirigindo sua reflexão e ação para a estruturação do cuidado em saúde e em enfermagem, que fosse demarcado pela construção coletiva, sempre multiprofissional e interdisciplinar, voltado à qualificação da vida.

\section{FORMAÇÃO DE RECURSOS HUMANOS E EXPRESSIVIDADE PARA ALÉM DA ENFERMAGEM E DO BRASIL}

Do ponto de vista acadêmico, Cecília contribuiu de forma significativa na formação de recursos humanos, nos âmbitos da graduação e da pós-graduação stricto sensu e lato sensu, em sintonia com as diretrizes e políticas emanadas dos Ministérios da Saúde e da Educação, bem como com as demandas do mercado de trabalho e dos serviços de saúde, evidenciando seu compromisso pessoal e profissional em prol da melhoria da qualidade da assistência à saúde da população. Nessa mesma base, fundamentou seus esforços na produção e divulgação do conhecimento.

A sua produtividade e desempenho acadêmico conduziram-na a posição de liderança no cenário nacional e internacional na área de Enfermagem, com ênfase em Enfermagem de Saúde Pública. Foi bolsista de Produtividade em Pesquisa do Conselho Nacional de Ciência e Tecnologia (CNPq), Nível 1B; foi consultora ad hoc de várias agências de fomento (CNPq, CAPES e FAPESP) e de 11 revistas nacionais e internacionais.

Foi Assessora Técnica junto ao Departamento de Gestão em Saúde do Ministério da Saúde, no ano de 2004, contribuindo para a discussão sobre as 
políticas voltadas à formação na área da saúde.

Cecília também teve um papel marcante junto a CAPES contribuindo de forma decisiva para consolidar a importância da Área de Enfermagem. Atuou desde 1990 assumindo várias funções:

1. 1990 a 2009 - Consultor ad hoc;

2. 1991 a 1993 - Presidente da Área de Enfermagem no Conselho Técnico Científico;

3. 1999 a 2001 - membro efetivo e consultor da Comissão de Avaliação Continuada dos Programas de Pós-Graduação da Área de Enfermagem;

4. 2006 a 2008 - membro efetivo do Comitê Multidisciplinar/Interdisciplinar responsável pela avaliação e pelo acompanhamento dos Programas de PósGraduação stricto sensu e pela Apreciação de Propostas de Cursos Novos (APCN) da referida área.

Essa última atuação evidencia que o reconhecimento da Profa. Cecília, na pós-graduação brasileira, transcende a área de enfermagem.

Demonstrou ampla experiência na formação de recursos humanos em enfermagem e saúde, tendo orientado 18 mestres, 22 doutores, três monografias de alunos de especialização, 15 alunos de iniciação científica e dois trabalhos de conclusão de curso de graduação, além de quatro orientações de outra natureza.

Ao longo de sua trajetória na EERP/USP, acumulou vasta experiência administrativa, seja no exercício da função de chefe e chefe suplente do Departamento de Enfermagem Materno-Infantil e Saúde Pública, Departamento que colaborou na sua fundação em 1985 e que ajudou a consolidar. Foi uma das responsáveis pela criação do Programa de Pós-Graduação Enfermagem em Saúde Pública, em 1991, o primeiro com característica multidisciplinar, e contribuiu com a sua consolidação desenvolvendo estudo sobre o produto científico do Programa e suas relações com os problemas oriundos das práticas de saúde, ${ }^{14}$ além de outras investigações e práticas vinculadas à linha de pesquisa "Práticas, saberes e políticas de saúde".

Ainda, sua experiência se voltou para outras instâncias, como membro de diversos colegiados e comissões assessoras, bem como na presidência da Comissão de Pós-Graduação da EERP/USP, na qual teve um papel de destaque para sua organização e consolidação. Ênfase especial merece ser dada a sua atuação à frente dessa Comissão, ao longo de 10 anos, período esse em que houve o fortalecimento dos três Programas de Pós-Graduação desta Unidade, o estabelecimento de diversas parcerias nacionais e internacionais, objetivando o desenvolvimento da pesquisa em Enfermagem no Brasil e na América Latina.

Da análise feita sobre a pós-graduação na EERP/USP, constatou impacto considerável na produção científica da área, permitindo elucidar várias lacunas do conhecimento da enfermagem e aprofundar muitas outras temáticas, garantindo o seu espaço junto aos pares da comunidade científica e possibilitando formar lideranças em pesquisa para várias regiões carentes de pós-graduação. Outros resultados apontados do ensino de pósgraduação da Unidade e que vêm contribuindo para a enfermagem brasileira são: consolidação dos grupos e núcleos de pesquisa na enfermagem; definição e estruturação das linhas de pesquisa; intercâmbio nacional e internacional de conhecimento científico de enfermagem com professores e pesquisadores; maior visibilidade da profissão de enfermagem no setor saúde e na sociedade; conquista de espaço participativo nas instituições científicas e tecnológicas nacionais. Esse patamar alcançado pela EERP aponta para a necessidade de projeção internacional, ${ }^{15}$ o que vem ocorrendo gradualmente nesses últimos cinco anos.

Cabe destacar a atuação de Cecília na divulgação dos cursos de mestrado e doutorado da EERP/USP nos países da América Latina, atraindo alunos da Argentina, Chile, Colômbia, Cuba, México, Peru e, ainda da África Portuguesa - de Angola. Ao liderar os processos de formação de recursos humanos por meio dos cursos de pós-graduação stricto sensu para universidades latino-americanas, seja por intermédio de acordos institucionais ou da utilização do Programa Estudante Convênio, contribuiu para a formação de massa crítica nessas Instituições, possibilitando a organização de núcleos de pesquisa e o surgimento de novos programas de pós-graduação em escolas e faculdades de enfermagem.

Coordenou dois projetos interinstitucionais firmado entre a USP, por meio da EERP, e a Universidade de Guanajuato (UGTO), através da Faculdade de Enfermagem e Obstetrícia de Celaya (FEOC), México, aprovados em 2001 e 2004, para a formação de doutores enfermeiros, docentes de Escolas e Faculdades de Enfermagem públicas do México, inscritos no Programa de Melhoramento do Profa.do (PROMEP) da Subsecretaria de Educação Superior e Investigação Científica, vinculada à Secretaria de Educação Pública do México. 
Um projeto de grande envergadura, que demonstrou a preocupação da Profa. Cecília com o incentivo e apoio a outros países da América Latina, com vistas a aumentarem o seu contingente de doutores em enfermagem, nucleando grupos de pesquisa, para futuramente criarem o doutorado e alavancarem a produção de conhecimento, ${ }^{16}$ refere-se ao México. Como fruto destes projetos acadêmicos operacionalizados nos períodos de março de 2002 a dezembro de 2004 e de março de 2005 a agosto de 2008, foram titulados 14 e 24 doutores em enfermagem, respectivamente.

Em continuidade ao compromisso social assumido para essa parceria com o México, sob a liderança da Profa. Cecília, há previsão da UGTO/ FEOC iniciar seu programa de doutoramento em enfermagem no $2^{\circ}$ semestre de 2009 e está em desenvolvimento um projeto de pesquisa com o objetivo de avaliar o impacto em atividades de ensino, pesquisa e extensão universitária, do doutorado em enfermagem vinculado aos convênios celebrados entre a USP/EERP e a UGTO/FEOC.

Vale destacar que este esforço no desenvolvimento da pós-graduação não se deu apenas no âmbito da EERP/USP. Merece ressaltar o talento inovador, liderança, abrangência social, trabalho incansável, integralidade e ética da Profa. Cecília em prol do desenvolvimento da pesquisa em Enfermagem na América Latina, comprovada por sua participação na Associação Latino-americana de Escolas e Faculdades de Enfermagem (ALADEFE), na Rede de Enfermagem da América Latina (REAL), na International Network of Doctoral Education Nursing (INDEN), (como membro da Comissão Temporária do Planning Committee, no período de 1999 a 2004), na coordenação de convênios de Cursos de Pós-Graduação Nível Doutorado, com o desejo de consolidar a enfermagem enquanto ciência no Brasil e na América Latina, por meio da formação de pesquisadores e de análise crítica sobre a enfermagem na dimensão da prática, do ensino, da produção de conhecimentos. ${ }^{17-23}$

Somem-se a isso, investigações em parceria com pesquisadores latino-americanos, publicações sobre o desenvolvimento de recursos humanos em saúde na América Latina e Brasil, a participação em eventos científicos para apresentação de trabalhos e discussão sobre o fortalecimento da pesquisa em enfermagem, ${ }^{24-26}$ visando contribuir com a produção de conhecimentos e de práticas na enfermagem, considerando a fundamentação teórica do processo de trabalho em saúde e enfermagem e o eixo da Atenção Primária à Saúde, privilegiando como objeto as Práticas, Saberes e Políticas de Saúde, com a abordagem de temáticas relacionadas ao processo de trabalho em enfermagem, a organização dos serviços de saúde e a formação de recursos humanos em saúde para o ensino, pesquisa e extensão.

Um último ponto a ser destacado, refere-se à sua contribuição na formação de pesquisadores no campo da Saúde Coletiva, utilizando-se da lógica de organização de grupos de pesquisa, sendo que, junto com a Profa. Semiramis Melani de Melo Rocha, e com a assessoria do Prof. Ricardo Bruno Mendes Gonçalves (docente da Faculdade de Medicina de São Paulo), criou e coordenou o Núcleo de Pesquisa e Estudos em Saúde Coletiva em 1989. Sob sua coordenação e da Profa. Semíramis, este Núcleo colaborou para a formação de pesquisadores de todo país, e contribuiu para o desenvolvimento de investigações de grande importância para a enfermagem brasileira, como por exemplo, o Projeto CIPESC - Classificação Internacional das Práticas em Saúde Coletiva, ${ }^{27-29}$ sob coordenação nacional da ABEn.

\section{CONCLUINDO}

Buscamos aqui, mesmo considerando a implicação dos autores com a Profa. Maria Cecília Puntel de Almeida, trazer um trajeto de história, de produção, de conhecimento, que se interligam em práxis. A idéia foi de relacionar a trajetória de um personagem importante da enfermagem brasileira - a Profa. Doutora Maria Cecília Puntel de Almeida - e entrelaçá-la com a história da enfermagem mais recente, apontando suas contribuições para a compreensão da enfermagem como trabalho.

Consideramos que a história das lideranças que compõem a enfermagem precisa ser demarcada, contada, transmitida, para que possamos num movimento de aprendizado e superação avançar nas possibilidades de (re)construção de uma prática de enfermagem mais qualificada, mais humana, mais ética, mais amorosa.

A história da Profa. Cecília pode nos ajudar a entender e construir estas possibilidades, uma vez que seu legado é produto e produtor do movimento de engajamento da enfermagem brasileira em lutas pela democracia e pela saúde como direito.

\section{REFERÊNCIAS}

5. Almeida MCP. Memorial apresentado para inscrição ao concurso de Professor Titular do Departamento de Enfermagem Materno-Infantil e Saúde Pública 
da Escola de Enfermagem de Ribeirão Preto da Universidade de São Paulo. Ribeirão Preto (SP): EERP-USP; 1992.

6. Almeida MCP, Rocha JSY. O saber de enfermagem e sua dimensão prática. São Paulo (SP): Cortez; 1986.

7. Almeida MCP, Mishima SM, Peduzzi M. A pesquisa em enfermagem fundamentada no processo de trabalho: em busca da compreensão e qualificação da prática de enfermagem. In: $51^{\circ}$ Congresso Brasileiro de Enfermagem; 1999. Florianópolis, Santa Catarina. Florianópolis: ABEn; 2000. p. 259-77.

8. Rocha SMM, Almeida MCP. O processo de trabalho da enfermagem em saúde coletiva e a interdisciplinaridade. Rev Latino-am Enfermagem. 2000 Nov-Dez; 8(6):96-101.

9. Almeida MCP, Rocha SMM. O Trabalho de enfermagem. São Paulo (SP): Cortez; 1997.

10. Almeida ES, Chioro A, Zioni F. Políticas públicas e organização dos serviços de saúde no Brasil e o sentido político do SUS. In: Westphal M, Almeida ES. organizadores. Gestão de serviços de saúde: descentralização, municipalização do SUS. São Paulo (SP): Editora da Universidade de São Paulo; 2001.p.13-50.

11. Relatório Final da Oitava Conferência Nacional de Saúde. In: Anais da $8^{\text {a }}$ Conferência Nacional de Saúde; 1986 Out 10-12; Brasília. Brasília (DF): Ministério da Saúde; 1987. p. 381-9.

12. Matus C. Política, planejamento e governo. Tomos I e II. Brasília (DF): Instituto de Pesquisa Econômica Aplicada; 1993.

13. Xavier IM, Souza AMA, Araujo EC, Rodriguez Neto E, Souza EO, Briceno G, et al. Subsídios para a conceituação da assistência de enfermagem rumo à Reforma Sanitária. Rev Bras Enferm. 1987 Abr-Set; 40(2-3):177-80.

14. Albuquerque GL, Pires DP. A construção de uma nova forma de representação profissional - um desafio no Projeto Político-Profissional da Enfermagem Brasileira. Rev Bras Enferm. 2006 MarAbr; 59(2):228-32.

15. Almeida MCP, Rodrigues AM, Castellanos BEP, Rosa MTL, Mendes SASA. A situação da enfermagem nos anos 80. In: Anais do $41^{\circ}$ Congresso Brasileiro de Enfermagem, 1989, Florianópolis. Anais. Florianópolis: Associação Brasileira de Enfermagem, 1989. p. 43-75.

16. Rosa MTL, Rodrigues AM, Castellanos BEP, Almeida MCP, Mendes SASA. O desenvolvimento técnico científico da enfermagem - uma aproximação com instrumentos de trabalho. In: Anais do $41^{\circ}$ Congresso Brasileiro de Enfermagem, 1989, Florianópolis. Anais. Florianópolis: Associação Brasileira de Enfermagem, 1989. p. 97-126.

17. Castellanos BEP, Rodrigues AM, Almeida MCP, Mendes SASA. Os desafios da enfermagem para os anos 90. In: Anais do $41^{\circ}$ Congresso Brasileiro de Enfermagem, 1989, Florianópolis. Anais. Florianópolis: Associação Brasileira de Enfermagem, 1989. p. 147-69.

18. Almeida MCP, Silva EM, Villa TCS, Assis MMA, Kemura MLR. O conhecimento produzido no programa de mestrado de enfermagem em saúde pública da Escola de Enfermagem de Ribeirão Preto e suas relações com as práticas de saúde. Rev Latinoam Enfermagem. 2000 Set-Out; 8(5):91-6.

19. Almeida MCP, Rodrigues RAP, Furegato ARF, Scochi CGS. A pós-graduação na Escola de Enfermagem de Ribeirão Preto - USP: evolução histórica e sua contribuição para o desenvolvimento da enfermagem. Rev Latino-am Enfermagem. 2002 Mai-Jun; 10(3):276-87.

20. Almeida MCP, Robazzi MLCC, Scochi CGS, Bueno SMV, Cassiani SHB, Saeki T, et al. Perfil da demanda dos alunos da pós-graduação stricto sensu da Escola de Enfermagem de Ribeirão Preto, da Universidade de São Paulo. Rev Latino-am Enfermagem. 2004 Mar-Abr; 12(2):153-61.

21. Angerami ELS, Almeida MCP. Divulgação do conhecimento científico produzido na enfermagem. Rev Esc Enferm USP. 1982 Ago; 16(2):108-27.

22. Almeida MCP, Barreira IA. Estudos de pós-graduação em enfermagem na América Latina: sua inserção na comunidade científica. In: Sena RR, organizadora. Educación de Enfermería en América Latina. Bogotá (CO): Universidad Nacional; 2000. p. 139-45.

23. Pagliuca LMF, Almeida MCP, Gutiérrez MGR, Erdmann AL, Leite JL, Kurcgant P. Critérios para classificar periódicos científicos de enfermagem. Acta Paul Enferm 2001 Set-Dez; 14(3):9-15.

24. Gutiérrez MGR, Vieira TT, Almeida MCP, Elsen I, Stefanelli MC. Acompanhamento e avaliação da pósgraduação no Brasil. Rev Enferm Esc Anna Nery. 2001 Ago; 5(2):161-72.

25. Almeida MCP, Gutiérrez MGR, Pagliuca LMF, Kurcgant P, Leite JL, Erdmann AL. Los programas de doctorado en América Latina: el estudio del caso de Brasil. In: Wright MG, Chisman AM, Gallegos EC, Bertha C, Gonzalez S, Ortega SE, et al organizadores. Los Nuevos Programas de Doctorado en Enfermería y su Contribución en la Reducción de la Demanda de Drogas en América Latina: Retos y Perspectivas. Washington, DC (US): Organización de los Estados Americanos - OEA; 2003. p. 127-47.

26. Rodrigues RAP, Erdmann AL, Almeida MCP. Los programas de postgrado (especialización, maestría y doctorado) - Brasil. In: Wright MG, Chisman AM, Padilha MICS, Gelbcke FL, Radünz V, Spricigo JS, Pires DEP, Souza ML. organizadores. La Situación de los Programas de Postgrado de Enfermería en Nueve Paises de América Latina Frente a los Desafios de la Reducción de la Demanda de Drogas. Washington (US): CICAD; 2003. p. 44-54. 
27. Pena YF, Almeida MCP, Duranza RLC. The Nursing Work Process in Care for Healthy Children at a Social Security Institution in Mexico. Rev Latino-am Enfermagem. 2006 Out; 14(5):651-7.

28. Silva GB, Almeida MCP, Ruffino MC, Steagall Gomes DL. Introdução à análise das transformações na prática de enfermagem no Brasil no período 1920-1978. Medicina 1984; 17(1-2):35-47.

29. Castro IB, Almeida MCP. La investigación en enfermeria como instrumento de cambio de la practica y en la formación de recursos humanos. Educ Med Salud. 1985; 19(3):313-36.

30. Chompré RR, Costa AE, Alves DB, Melo FNP, Teixeira JS, Mendes LC, et al. Relatório do seminário de viagens de enfermeiras docentes-assistenciais que atuam em programas de assistência primária/ Trips seminary report of teaching-care nurses whom act in primary care programs. Belo Horizonte (MG): Universidade Federal de Minas Gerais; 1986.

31. Antunes MJM, Silva IA, Egry EY, Sena RR, Almeida MCP. Projeto de classificação das práticas de enfermagem em saúde coletiva - Brasil - manual do pesquisador: orientação para o trabalho de Campo. Brasília (DF): Associação Brasileira de Enfermagem; 1997.

32. Egry EY, Antunes MJM, Sena RR, Almeida MCP, Silva IA. Classification of nursing practice en collective health: the Brazilian experience to classify - CIPESC/ICN/ABEn. In: Chianca TCM, Antunes MJM, organizadores. International Classification of Nursing Practice in Collective Health in Brazil CIPESC/Brazil. Brasília (DF): Associação Brasileira de Enfermagem; 1999. p.11-22.

33. Almeida MCP, Palha PF, Domingos MMLN, Mishima SM, Villa TCS, Oba MDV. A classificação internacional da prática de enfermagem em saúde coletiva no Brasil - CIPESC/ICN/ABEn e o processo de trabalho. In: Chianca TCM, Antunes MJM, organizadores. A classificação Internacional das Práticas de Enfermagem em Saúde Coletiva - CIPESC - Série Didática: enfermagem no SUS. Brasília (DF): Associação Brasileira de Enfermagem; 1999. p.46-56. 